\title{
APLICAÇÃO DE AGENTES QUÍMICOS DE CRESCIMENTO EM BOLO DE CHOCOLATE
}

\author{
Krischina Singer APLEVICZ ${ }^{1}$ \\ Fabíola Zimmermann SCHMITZ ${ }^{2}$ \\ Luiza Ferrazza DIAS ${ }^{3}$
}

1 Doutora em Ciência dos Alimentos, Professora do Instituto Federal de Educação, Ciência e Tecnologia de Santa Catarina, aplevicz@gmail.com

2 Especialista em Gastronomia, Planejamento e Gestão Estratégica, Professora do Instituto Federal de Educação, Ciência e Tecnologia de Santa Catarina, fabiolaz@ifsc.edu.br

${ }^{3}$ Aluna bolsista do curso Técnico em Panificação do Instituto Federal de Educação, Ciência e Tecnologia de Santa Catarina, luizafdias@gmail.com

Recebido em: 16/05/2014 - Aprovado em: 30/06/2014 - Disponibilizado em: 30/07/2014

RESUMO: O bolo é um alimento comumente consumido pelas pessoas de idades diversas. Consiste em uma mistura de gordura e açúcar, com mais outros ingredientes. Em algumas formulações é necessário o uso de agentes químicos de crescimento. O objetivo deste estudo foi aplicar agentes químicos de crescimento, como fermento químico, bicarbonato de sódio e a mistura de ambos em bolos de chocolate. Foram realizadas a análise de volume específico e teste de aceitabilidade com cinqüenta julgadores não treinados. Todos os bolos foram aceitos na análise sensorial. A amostra com fermento químico apresentou a maior nota no quesito sabor e a menor no atributo cor. O uso do bicarbonato de sódio ocasionou escurecimento da massa do bolo.

Palavras-chave: Bolo. Expansão. Aceitabilidade.

ABSTRACT: The cake is a food commonly consumed by people of different ages. It consists of a mixture of fat and sugar, with over other ingredients. In some formulations the use of chemical growth is required. The aim of this study was to analyze chemical growth agents such as baking powder, bicarbonate of soda and mix of both in chocolate cakes. The analysis of specific volume and acceptability fifty untrained judges test were performed. All cakes were accepted in sensory analysis. The sample with baking powder showed the highest and the lowest note in the taste Question on color attribute. The use of sodium bicarbonate of soda caused darkening of the cake dough.

Keywords: Cake. Expansion. Acceptability.

\section{INTRODUÇÃO}

Bolo é uma mistura de farinha de qualquer tipo, acrescido de algum tipo de gordura, açúcar e ovos, e levado ao forno para assar (GOMENSORO, 1999). É considerado a segunda categoria de produtos que motivam a compra nas padarias, perdendo somente para o pão (CHUDZIKIEWICZ, 2005). Muitas empresas que já atuavam com pães, biscoitos e torradas ingressaram no mercado de bolos a fim de diversificar sua linha de produtos (PAVANELLI et al., 2000). 
A qualidade do bolo é determinada por características essenciais, como: textura macia, que deve permanecer inalterada ao longo da vida de prateleira do produto, superfície uniforme, homogeneidade do miolo, volume adequado, palatabilidade, sabor agradável e facilidade de processamento. Estes atributos estão diretamente relacionados à qualidade dos ingredientes e ao seu balanceamento (PAVANELLI et al., 2000).

Massas de bolo são compostas por um sistema bifásico: gorduras e açúcares e os outros ingredientes da mistura. A fase gordurosa se apresenta dispersa, de forma irregular, com partículas de forma e tamanhos variados. Internamente, nas partículas de gordura são encontradas inúmeras bolhas de ar, que são incorporadas durante o processo de mistura e batimento (ESTELLER et al., 2006a). Ao ser levada ao forno a massa deve crescer lenta e uniformemente, processo que se obtém pela ação do calor sobre as partículas de gás carbônico $\left(\mathrm{CO}_{2}\right)$ que se expandem à medida que são liberadas pela reação química do fermento (KAJISHIMA; BERNARDI, 2005).

As massas duras e moles crescem com o poder do vapor de água, ar e gás carbônico ou por uma mistura desses três elementos. $\mathrm{O}$ ar é composto por uma mistura de gases e é incorporado durante o batimento, como no caso das claras em neve ou no bater da gordura e da massa. O vapor é formado pela evaporação da água ao assar da massa e o gás carbônico é formado através da reação química que ocorre pela ação entre o bicarbonato de sódio e um ácido (SINO; CIANCIULLI, 2008). A quantidade de fermento em pó a ser utilizada depende do tipo de bolo, das características e quantidades dos ingredientes empregados e da altitude, além do modo de misturar e manipular a massa (VITTI et al.,1988).

Em muitas preparações, somente a produção de vapor ou a incorporação de ar não são suficientes para o crescimento total das massas, indicando a necessidade de outro tipo de agente de crescimento (ARAÚJO, et al., 2011). O fermento químico em pó é um agente fermentador químico adicionado à mistura ou à massa para causar expansão. A ação fermentadora é causada pela interação de uma substância alcalina, bicarbonato de sódio e ingredientes ácidos, como cremor de tártaro ou sulfato sódico de alumínio, os quais causam a eliminação do gás carbônico dentro da massa (CANELLA-RAWLS, 2005).

O bicarbonato de sódio é comumente usado como expansor em misturas rápidas, por si mesmo não oferece ação fermentadora, pois precisa ser ativado pela presença de um ácido e um líquido (CANELLA-RAWLS, 2005). Quando aquecido forma gás carbônico, carbonato de sódio $\left(\mathrm{Na}_{2} \mathrm{Co}_{3}\right)$ e água. Quando somente o bicarbonato é utilizado como agente de crescimento, o resíduo $\mathrm{Na}_{2} \mathrm{Co}_{3}$ permanece no bolo (VITTI et al., 1988). 
O objetivo do presente trabalho foi verificar o efeito, em bolos de chocolate, de agentes químicos de crescimento como fermento químico, bicarbonato de sódio e a combinação de ambos.

\section{MATERIAL E MÉTODOS}

Elaboração dos bolos

A formulação dos bolos de chocolate está ilustrada na Tabela 1. A massa foi misturada em batedeira (KitchenAid) por 20 minutos. Primeiro foi adicionada as gemas com o açúcar até formar um creme branco. Em seguida foi adicionado o óleo de soja, o cacau em pó, a água fervente e a farinha de trigo para, em seguida, adicionar as claras em neve e o agente de crescimento. A massa foi moldada em formas retangulares e assada em forno turbo (Líder) a $160^{\circ} \mathrm{C}$ por $35 \mathrm{~min}$. Estes equipamentos estão disponíveis no Laboratório de Confeitaria do Campus Florianópolis-Continente do Instituto Federal de Educação, Ciência e Tecnologia de Santa Catarina.
Tabela 1- Formulação em porcentagem utilizada na elaboração dos bolos de chocolate

\begin{tabular}{lccc}
\hline \multicolumn{1}{c}{ Ingredientes } & A & B & C \\
\hline Açúcar refinado & 100 & 100 & 100 \\
Ovos & 83 & 83 & 83 \\
Farinha de trigo & 67 & 67 & 67 \\
Água & 67 & 67 & 67 \\
Óleo de soja & 25 & 25 & 25 \\
Cacau em pó & 22 & 22 & 22 \\
Fermento químico & 5 & 0 & 2,5 \\
Bicarbonato de sódio & 0 & 5 & 2,5 \\
\hline \multicolumn{2}{c}{ Fonte: Autores. } & & \\
Legenda: & & \\
A - Fermento químico & & \\
B - Bicarbonato de sódio & \\
C - Fermento químico e bicarbonato de sódio &
\end{tabular}

Análise do volume específico dos bolos

$$
\mathrm{Na} \text { obtenção das massas para }
$$
avaliação da propriedade de expansão, dividiu-se a massa em três partes com pesos iguais, sendo levadas a um forno pré-aquecido a $180^{\circ} \mathrm{C}$ e assadas por 25 minutos. As esferas expandidas e resfriadas foram pesadas, impermeabilizadas com parafina fundida e seus volumes medidos pelo deslocamento de água em proveta graduada. O resultado da expansão foi expresso em volume específico, em mL/g ${ }^{-1}$ (DEMIATE; CEREDA, 2000).

Análise sensorial dos bolos

A análise sensorial dos produtos foi realizada por meio do teste de aceitabilidade (FARIA; YOTSUYANAGI, 2000). Cada amostra foi testada por um grupo de cinqüenta julgadores não-treinados $(n=50)$, utilizando a escala hedônica estruturada de nove pontos (1 - desgostei extremamente; 9 - gostei extremamente). Para as amostras analisadas no teste de aceitabilidade, foram consideradas 
aceitas quando mais de $50 \%$ dos provadores apontaram valores maiores ou iguais a seis, que corresponde ao menor grau de gostar, segundo a escala hedônica (SILVA et al., 2003). Foi aplicado um questionário para se obter informações sobre os julgadores.

\section{Análise estatística}

Os resultados foram analisados utilizando o programa Statistica ${ }^{\circledR}$ versão 8.0 (Statsoft Inc., Tulsa, OK, USA). As diferenças entre as médias foram calculadas através da análise de variância one-way (ANOVA) com o teste de Tukey. Foram consideradas significativas as diferenças ao nível de $5 \%$ (P $<0,05)$.

\section{RESULTADOS E DISCUSSÃO}

Características dos bolos

Na Figura 1 está ilustrado os bolos elaborados com os diferentes agentes químicos de crescimento. Os diferentes tipos de fermentos não influenciaram o volume específico das amostras $(\mathrm{P}>0,05)$. A amostra com fermento químico apresentou maior volume específico, porém não diferente significativamente das demais (Tabela 2).

Figura 1 - Bolos de chocolate com diferentes agentes químicos de crescimento

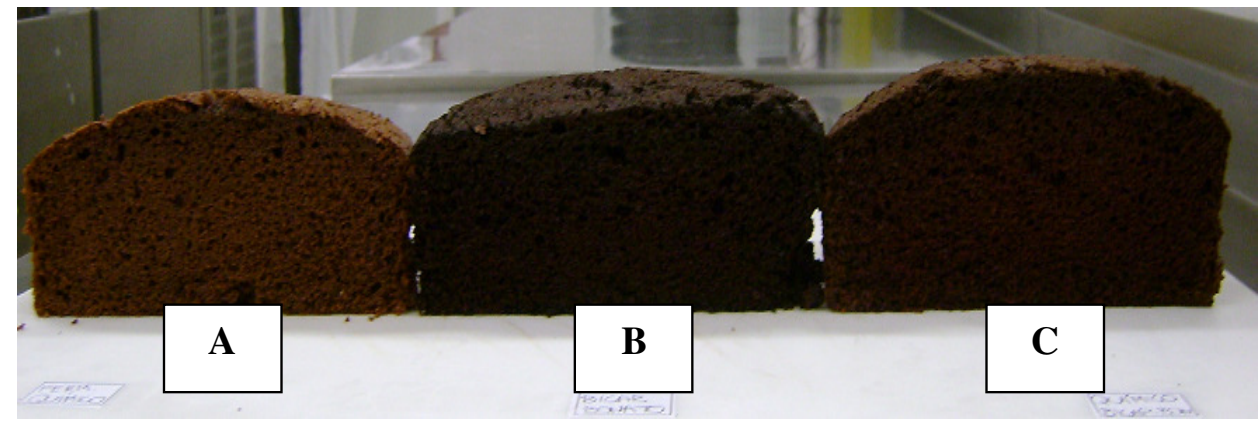

Fonte: Autores.

Legenda:

A - Fermento químico

B - Bicarbonato de sódio

C - Fermento químico e bicarbonato de sódio

O volume específico e a densidade mostram claramente a relação entre o teor de sólidos e a fração de ar existente na massa assada (ESTELLER et al., 2005). A qualidade dos bolos está diretamente relacionada ao tipo de processamento e à qualidade e balanceamento correto dos ingredientes (PAVANELLI et al., 2000). A formação de estrutura uniforme dos alvéolos é importante para a qualidade de massas assadas (ESTELLER et al., 2006a). Os valores de volume específico neste estudo foram maiores que os obtidos por Esteller et al. (2006b) que elaborou um bolo tipo inglês 
substituindo cacau em pó por pó de cupuaçu e kefir.

Tabela 2 - Volume específico das amostras de bolo de chocolate coloração escura e sabor desagradável (VITTI et al., 1988). Quando adicionados fermento químico e bicarbonato de sódio em quantidade elevada podem conferir ao produto sabor amargo, adstringente ou de sabão (ARAÚJO et al., 2011).

O cacau em pó fornece sabor e cor Volume específico $3,57^{\mathrm{a}} \pm 0,10 \quad 3,09^{\mathrm{a}} \pm 0,12 \quad 3,08^{\mathrm{a}} \pm 0,02$ aos produtos de panificação e confeitaria, mas * médias com letras iguais não diferem entre si estatisticamente $(P>0,05)$.

Fonte: Autores. Legenda: A - Fermento químico B - Bicarbonato de sódio C - Fermento químico e

bicarbonato de sódio

As notas do teste de aceitabilidade estão apresentadas na Tabela 3. No que tange à coloração, o bolo preparado com fermento químico obteve a menor nota sensorial. A maior nota sensorial foi para o bolo com fermento químico e bicarbonato de sódio na mesma formulação $(\mathrm{P}<0,05)$. Na Figura 1 é possível observar que o bolo com bicarbonato de sódio apresentou coloração mais escura quando comparada a com fermento químico. ARAÚJO et al. (2011) afirmaram que em bolos de chocolate costuma-se acrescentar além do fermento químico, o bicarbonato de sódio para obter a cor mais escura da massa assada.

A amostra preparada com fermento químico obteve a melhor nota no atributo sabor e a com ambos os agentes de crescimento obteve a menor nota $(\mathrm{P}<0,05)$. Em excesso, o bicarbonato de sódio reage com a gordura presente na receita, causa a sua adição tende a aumentar ligeiramente o $\mathrm{pH}$ dos produtos ligeiramente ácidos. Nos produtos da confeitaria, a alcalinidade pode ser reforçada mediante a adição de uma quantidade extra de bicarbonato de sódio na receita, o que intensifica a cor do chocolate (CAUVAIN; YOUNG, 2008).

Com relação a aparência, não foi observado diferença significativa entre os tratamentos $(\mathrm{P}>0,05)$. A adição de cacau, fibras, amidos, frutas secas é crítica, pois pode deixar o produto com aparência "embatumada", isto é maior crescimento na parte superior e massa úmida e compacta na parte inferior (JOOSTE, 1951).

Todas as amostras foram consideradas aceitas nos atributos sabor, cor e aparência, pois mais de $50 \%$ dos provadores apontaram valores maiores ou iguais a 6 , que corresponde ao menor grau de gostar (SILVA et al., 2003). A valorização das características dos produtos alimentícios iniciam com a observação visual da aparência física e segue com a apreciação do aroma ou odor, da textura e do sabor (CAUVAIN; YOUNG, 2008). 
Tabela 3 - Médias do teste de aceitabilidade

\begin{tabular}{cccc}
\hline Atributos & A & B & C \\
\hline Cor & $6,52^{\mathrm{a}}$ & $7,16^{\mathrm{a}, \mathrm{b}}$ & $7,60^{\mathrm{b}}$ \\
Sabor & $7,16^{\mathrm{a}}$ & $7,08^{\mathrm{a}, \mathrm{b}}$ & $6,46^{\mathrm{b}}$ \\
Aparência & $7,22^{\mathrm{a}}$ & $6,22^{\mathrm{a}}$ & $7,11^{\mathrm{a}}$ \\
\hline
\end{tabular}

*Médias com letras iguais, na mesma linha, não diferem entre si estatisticamente $(P>0,05)$.

Fonte: Autores

Legenda:

A - Fermento químico

B - Bicarbonato de sódio

C - Fermento químico e bicarbonato de sódio

Do total dos provadores, $58 \%$ foram mulheres. Com relação a faixa etária, foi observado que $20 \%$ foram pessoas com até 20 anos; $42 \%$ foram pessoas de 21 a 35 anos; $18 \%$ de 36 a 50 anos e $20 \%$ acima de 51 anos. Com relação ao consumo diário de bolos, $56 \%$ relataram que consomem uma vez na semana e $26 \%$ duas vezes. Na Figura 2 é possível verificar o consumo de bolos, sendo podendo ser observado que as mulheres são as maiores consumidoras de bolos. Embora não constitua alimento básico como o pão, o bolo é aceito e consumido por pessoas de qualquer idade (BORGES et al., 2006).

Figura 2 - Consumo semanal de bolo

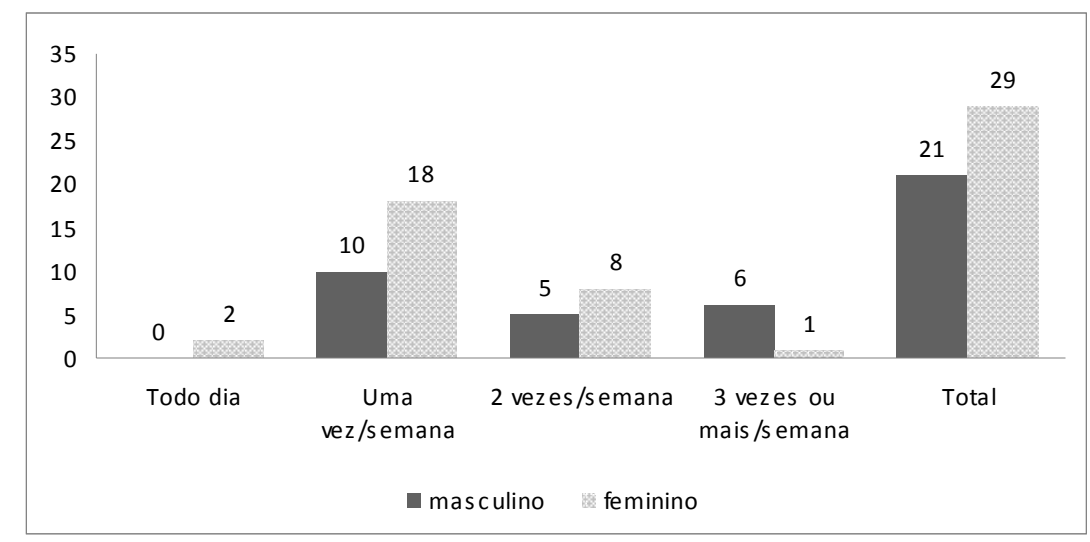

Fonte: Autores.

\section{CONSIDERAÇÕES FINAIS}

Apesar de todos os bolos terem sido aceitos na análise sensorial, observa-se que o preparado com fermento químico apresentou a maior nota no quesito sabor e a menor no atributo cor. $\mathrm{O}$ uso do bicarbonato de sódio ocasionou escurecimento da massa do bolo. Não foi observada diferença estatística entre as amostras com relação ao volume específico e aparência.

\section{REFERÊNCIAS}

ARAÚJO, W.M.C.; MONTEBELLO, N. di P.; BOTELHO, R.B.A.; BORGO, L.A. Alquimia dos alimentos. Editora Senac: Brasília, 2011.

BORGES, J.T. da S.; PIROZI, M.R.; LUCIA, S.M.D.; PEREIRA， P.C.; FIALHO E MORAES, A.R.; CASTRO, V.C. Utilização de farinha mista de aveia e trigo na elaboração 
de bolos. Boletim Ceppa, v. 14, n.1, p.145$162,2006$.

CANELLA-RAWLS, S.C. Pão, arte e ciência. São Paulo: Senac, 2005.

CAUVAIN, S.; YOUnG, L. Productos de Panadería: ciência, tecnologia y práctica. Espana: Zaragoza, 2008.

CHUDZIKIEWICZ, F. F. Análise do comportamento de compra e de satisfação do cliente no mercado de panificadoras e confeitarias em Curitiba. Curitiba, 2005. 225 p. Dissertação (Mestrado em administração) - Centro de Ciências Sociais Aplicadas, Pontifícia Universidade Católica do Paraná, 2005;

DEMIATE, I. M.; CEREDA, M. P. Some physico-chemical characteristics of modified cassava starches presenting baking property. Energia na Agricultura, v. 15, n. 3, p. 36-46, 2000.

ESTELLER, M.S.; LANNES, S.C.S. Parâmetros complementares para fixação de identidade e qualidade de produtos panificados. Revista Ciência e Tecnologia de Alimentos, v. 25, n.4, p.802-806, 2005.

ESTELLER, M.S.; ZANCANARO JÚNIOR, O.; LANNES, S.C.S. The effect of kefir addition on microstructure parameters and physical properties of porous white bread.
Europe Food Research Technology, v. 222, p. 26-31, 2006a.

ESTELLER, M.S.; ZANCANARO JÚNIOR, O.; LANNES, S.C.da S. Bolo de chocolate produzido com pó de cupuaçu e kefir. Brazilian Journal of Pharmaceutical Sciences, v. 42, n. 3, $2006 \mathrm{~b}$.

FARIA, E. V. de; YOTSUYANAGI, K. Técnicas de análise sensorial. Campinas: ITAL/ LAFISE, 2002.

GOMEnSORO, M. L. Pequeno dicionário de gastronomia. Rio de Janeiro: Objetiva, 1999.

JOOSTE, M.E. Cake structure and palability as affected by emulsifysing agents and baking temperatures. Oregon State College. 1951.

KAJISHIMA， S.; BERNARDI， M.R.V. Técnica Dietética. São Paulo, 2005.

PAVANELLI, A.P.; CICHELLO, M.S.; PALMA, E.J. Emulsificantes como agentes de aeração em bolos. 2000. Disponível em $<$ http://www.oxiteno.com.br>. Acesso em: 12 Dez. 2011.

SILVA， M.E.M.P.e; YONAMINE; G.H.; MITSUKI, L. Desenvolvimento e avaliação de pão francês caseiro sem sal. Brazilian Journal Food Technology, v.6, n.2, p. 229236, 2003. 
SINO, I.K.; CIANCIULLI, G. Agente de crescimento para bolos industrializados e mistura de bolos. Revista Aditivos e Ingredientes, n.55, p.66-68, 2008.

VITTI, P.; GARCIA, E.E.C.; OLIVEIRA.

Tecnologia de Biscoitos. Campinas, 1988. 